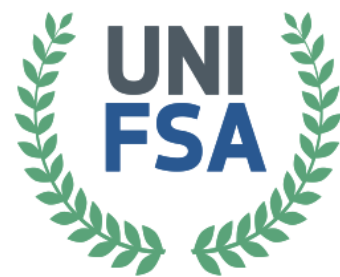

CENTRO UNIVERSITÁRIO SANTO ACOSTINHO

CENTRO UNIVERSITÁRIO SANTO ACOSTINHO

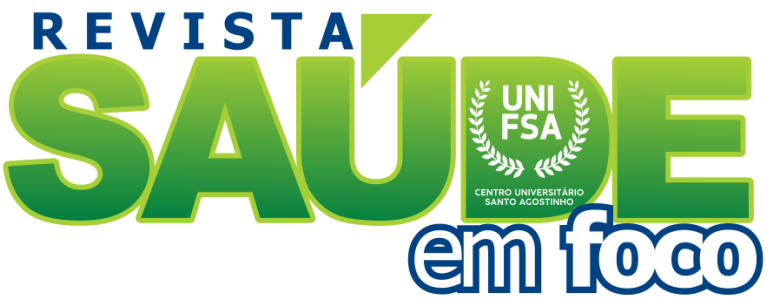

www4.fsanet.com.br/revista

Revista Saúde em Foco, Teresina, v. 8, n. 3, art. 3, p. 39-53, set./dez. 2021

ISSN Eletrônico: 2358-7946

http://dx.doi.org/10.12819/rsf.2021.8.3.3

\title{
Alergia Alimentar: Um Problema Crescente
}

Food Allergy: A Growing Problem

Mariana Alves dos Santos

Discente do Curso de Nutrição pela Universidade Estácio de Sá

Email: mariana.s.alves.ma@gmail.com

Letícia Telles Pereira Montes

Nutricionista pela Universidade Estácio de Sá

Email: leticiatellessh@gmail.com

Francine Albernaz Teixeira Fonseca Lobo

Doutora em Ciências Aplicadas a Produtos para Saúde pela Universidade Federal Fluminense Professora e Bolsista Produtividade da Universidade Estácio de Sá

Email: francinealbernazlobo@gmail.com

Endereço: Mariana Alves dos Santos

Rua Bingen, 50 - Bingen, Petrópolis - 25660-004, RJ Brasil.

Endereço: Letícia Telles Pereira Montes

Rua Bingen, 50 - Bingen, Petrópolis - 25660-004, RJ,

Brasil.

Endereço: Francine Albernaz Teixeira Fonseca Lobo Rua Bingen, 50 - Bingen, Petrópolis - 25660-004, RJ, Brasil.
Editor-Chefe: Dr. Tonny Kerley de Alencar Rodrigues

Artigo recebido em 22/01/2021. Última versão recebida em 20/10/2021. Aprovado em 21/10/2021.

Avaliado pelo sistema Triple Review: a) Desk Review pelo Editor-Chefe; e b) Double Blind Review (avaliação cega por dois avaliadores da área).

Revisão: Gramatical, Normativa e de Formatação 


\title{
RESUMO
}

Esse artigo teve como objetivo avaliar a prevalência das alergias alimentares e identificar os alimentos com maior incidência de alergias alimentares. Foram utilizados artigos científicos na língua portuguesa e inglesa, no período de 2011 a 2019. A alergia alimentar é uma reação clínica adversa desencadeada pela ingestão de alimentos alergênicos, mediados por uma resposta imunológica anormal, mediados por IgE, ou seja, qualquer substância capaz de estimular uma resposta de hipersensibilidade entre alergênicos. A intolerância alimentar caracteriza-se por qualquer reação adversa a um alimento envolvendo uma resposta não imunitária. Essa pode ser de origem enzimática, farmacológica ou indeterminada. A dieta de eliminação é a forma comprovadamente eficaz nos tratamentos das alergias alimentares e o tratamento mais promissor que está sendo investigado é o uso de imunoterapia antígenoespecífica, que consiste no fornecimento de quantidade crescente de alérgeno por um longo período de tempo, porém, há necessidade de mais estudos.

Palavras Chaves: Alergia Alimentar. Intolerância Alimentar. Food Allergy.

\begin{abstract}
This article aimed to assess the prevalence of food allergies and identify foods with the highest incidence of food allergies. Scientific articles were used, in Portuguese and English, between 2011 and 2019. Food allergy is an adverse clinical reaction triggered by the ingestion of allergenic foods, mediated by an abnormal immune response, mediated by $\operatorname{IgE}$, that is, any capable substance to stimulate a hypersensitivity response among allergens. Food intolerance is characterized by any adverse reaction to a food involving a non-immune response. This can be of enzymatic, pharmacological or undetermined origin. The elimination diet is the proven effective form in the treatment of food allergies and the most promising treatment under investigation is the use of antigen-specific immunotherapy, which consists of providing an increasing amount of allergen for a long period of time, but there is more need of studies.
\end{abstract}

Keywords: Alergia Alimentar. Intolerância Alimentar. Food Allergy. 


\section{INTRODUÇÃO}

A alergia alimentar é cada vez mais reconhecida como um problema crescente para a saúde pública. As evidências atuais sugerem que as alergias alimentares afetam cerca de $10 \%$ das crianças em alguns países, com crescente evidência estatística nas últimas décadas. Esses aumentos na prevalência afetaram preferencialmente as regiões industrializadas, embora agora haja também evidências crescentes de aumento da prevalência em países em rápido desenvolvimento, proporcional ao crescimento econômico crescente (LOH, W; TANG, ML, 2018).

A alergia alimentar tem sido associada ao estilo de vida moderno, podendo ter fatores genéticos ou ambientais envolvidos. Esse tipo de alergia possui uma diversidade de fatores de risco que pode influenciar seu surgimento, que incluem gênero, raça/etnia, genética, atopia, fatores nutricionais (insuficiência de vitamina D, gordura dietética, consumo reduzido de antioxidantes, obesidade) incluindo mudanças nos padrões alimentares, mudando bactérias comensais intestinais e poluição veicular (PRESCOTT, S., et al., 2011).

Ainda não se sabe se o aumento da alergia alimentar é um prenúncio de efeitos anteriores e mais graves dessas mudanças ambientais progressivas, ou se fatores de estilo de vida adicionais ou não relacionados estão implicados. Novos estudos sugerem que fatores ambientais podem produzir mudanças epigenéticas na expressão gênica e risco de doenças que podem ser potencialmente hereditárias ao longo das gerações (PRESCOTT, S., et al., 2011).

A alergia alimentar é definida como uma reação adversa imunomediada aos alimentos. De acordo com os mecanismos fisiopatológicos envolvidos, as reações adversas a alimentos podem ser classificadas em imunológicas, reações de hipersensibilidade imediata mediadas por IgE, ou não-imunológicas, reações tardias não mediadas por IgE e distúrbios com contribuições de vias imunológicas mediadas por IgE e não mediadas por IgE (TORDESILLAS, L. et al.2017).

A Agência Nacional de Vigilância Sanitária (ANVISA) já identificou mais de 170 alimentos como alergênicos. No entanto, cerca de $90 \%$ dos casos de alergia alimentar são ocasionados por apenas oito alimentos que são: ovos, leite, peixe, crustáceos, castanhas, amendoim, trigo e soja.

Portanto, sabendo-se que a alergia alimentar é causada, na maioria das vezes, por algum alimento especifico, e que várias pesquisas estão ampliando a perspectiva para esse 
campo, torna-se pertinente avaliar a prevalência da alergia alimentar e identificar os alimentos com maior incidência desse tipo de alergia. Avaliar as formas de diagnóstico e tratamento através da análise de artigos, através de uma revisão da bibliografia com o foco direcionado à alergia alimentar.

\section{METODOLOGIA}

O trabalho realizado trata-se de uma revisão integrativa da literatura sobre Alergia Alimentar, cuja finalidade é reunir, avaliar criticamente, identificar a aplicabilidade e condensar os resultados dos estudos incluídos.

O estudo foi conduzido no período de Março de 2021 a Agosto de 2021, por meio da busca de publicações da literatura científica, a partir das bases de dados: PUBMED, Scientific Eletronic Library Online (SciELO), LILACS e Portal de Periódicos da CAPES.

Os artigos consultados para integrarem a revisão foram selecionados de acordo com o tema e a utilização dos descritores: "Alergia Alimentar", "Intolerância Alimentar", "Food Allergy".

Os artigos foram selecionados levando em consideração seu período de publicação, fazendo parte da inclusão apenas estudos que tivessem sido publicados no período de 2011 a 2019.

\subsection{Epidemiologia}

Estima-se que 1/4 da população mundial irá enfrentar uma reação adversa a alimento durante a vida. Por isso, esse tema merece atenção por parte da população e da mídia, visto que uma manifestação clínica bem grave relacionada à Alergia Alimentar (AA) é a anafilaxia. (BERZUINO, M. et al. 2017).

As estatísticas sobre a alergia alimentar são conflitantes e variáveis, dependendo de fatores como: idade e características da população estudada (cultura, hábitos alimentares, clima), mecanismo imunológico envolvido, método de diagnóstico (autorreferido, questionário escrito, testes cutâneos, determinação de $\operatorname{IgE}$ sérica específica ou testes de provocação oral), tipo de alimento, regiões geográficas, entre outros. A AA se torna mais comum em crianças e a sua prevalência parece ter aumentado nas últimas décadas em todo o mundo. Estima-se que a prevalência seja aproximadamente de $6 \%$ em menores de três anos, e de 3,5\% em adultos (Consenso brasileiro sobre alergia alimentar, 2018). 
A alergia causada pelo leite de vaca, ovo, trigo e soja costuma desaparecer ainda na infância; em relação ao amendoim, nozes e frutos do mar, a alergia pode durar a vida toda. Em crianças de até dois anos de idade a proteína do leite de vaca é o alérgeno mais frequentemente observado, afetando de 2 a $3 \%$ das crianças no primeiro ano de vida. Crianças que possuem AA são até 4 vezes mais propensas a desenvolver outra condição relacionada, como a asma, em relação a crianças que não possuem AA. Cerca de 4 a $8 \%$ das crianças com asma possuem AA. Outro dado importante revela que aproximadamente $35 \%$ das crianças com dermatite atópica apresentam AA mediada por IgE. De acordo com um estudo realizado em 2013 pelo Centers for Disease Control and Prevention nos EUA, a AA aumentou cerca de 50\% em crianças no período entre 1997 e 2011. Mesmo diante do notável aumento dos casos no Brasil, até o momento não há dados precisos sobre a prevalência dessa hipersensibilidade alimentar (BERZUINO, M. et al. 2017).

Estudo norte-americano avaliou a prevalência de alergia alimentar na população geral (questionário online), porém, pode-se observar uma oscilação entre $1 \%$ e abaixo de $10 \%$; estudo posterior em crianças e baseado no relato de seus pais apontou ser de $8 \%$. Todo estudo populacional realizado a partir de registros médicos eletrônicos integrados do Partners HealthCare, em Boston (EUA), avaliou os dados sobre reações adversas a alimentos, entre 2000 e 2013. Foram avaliadas as reações potencialmente mediadas por IgE e anafiláticas entre 27 milhões de pacientes. Identificou-se ser a prevalência de intolerância/alergia a pelo menos um alimento 3,6\% (SOLÉ, D, et al. 2018).

\begin{tabular}{|c|c|c|}
\hline Alimento & Principais proteínas & \begin{tabular}{|l|} 
Principais sintomas \\
\end{tabular} \\
\hline Leite de vaca & $\begin{array}{c}\text { Caseína } \\
\alpha \text {-lactoalbúmina } \\
\text { B-lactoglobulina }\end{array}$ & $\begin{array}{l}\text { Urticária, angiodema, eritema, } \\
\text { dermatite atópica; dor abdominal, } \\
\text { náuseas, vômitos, diarreias; } \\
\text { prurido e edema de lábios, língua } \\
\text { e garganta, obstrução nasal, } \\
\text { espirros, dispneia, asma e } \\
\text { anafilaxia. }\end{array}$ \\
\hline Ovo & $\begin{array}{l}\text { Ovoalbumina } \\
\text { Ovomucóide } \\
\text { Conalbumina }\end{array}$ & $\begin{array}{l}\text { Urticária, angiodema, dermatite } \\
\text { atópica; asma, rinoconjutivite; dor } \\
\text { abdominal, náuseas, vômitos, } \\
\text { diarreias; hipotensão, choque, } \\
\text { anafilaxia. }\end{array}$ \\
\hline
\end{tabular}




\begin{tabular}{|c|c|l|}
\hline Trigo & Gliadina, glutenina & $\begin{array}{l}\text { Urticária,irritação na pele ou perda de peso, } \\
\text { irritação na garganta, choques anafiláticos. }\end{array}$ \\
\hline Soja & Gly $\mathbf{m}$ 5 & $\begin{array}{l}\text { Urticária, angiodema; dor } \\
\text { abdominal, náuseas, diarreia, } \\
\text { vômitos e dificuldade no ganho } \\
\text { de peso }\end{array}$ \\
\hline Amendoim & Ara h 1 & $\begin{array}{l}\text { Urticária, angiodema; edema } \\
\text { laríngeo, tosse, mudança na voz, } \\
\text { broncoespasmo; diarreia e } \\
\text { vômitos. }\end{array}$ \\
\hline Frutos do mar 3 & Tropomiosina & $\begin{array}{l}\text { Prurido, conjuntivite, rinite, asma, } \\
\text { urticária, angiodema, náuseas, } \\
\text { vômitos e anafilaxia. }\end{array}$ \\
\hline
\end{tabular}

\section{RESULTADOS E DISCUSSÕES}

\subsection{Alergia alimentar}

É importante distinguir Alergia Alimentar de Intolerância Alimentar. A AA é uma reação clínica adversa desencadeada pela ingestão de alimentos alergênicos, mediados por uma resposta imunológica anormal, mediados por IgE, ou seja, qualquer substância capaz de estimular uma resposta de hipersensibilidade entre alergênicos. São, na sua maioria, representados por glicoproteínas hidrossolúveis. As reações ocorrem quando o indivíduo sensível entra em contato com alimento alergênico, as células brancas do sistema imunológico (linfócitos B) produzem anticorpos específicos conhecidos como IgE, que ligam-se por meio dos receptores de alta afinidade aos mastócitos, assim, quando os mastócitos entrarem em contato com os alergênicos, iniciam uma resposta imune mais complexa, envolvendo a liberação de mediadores inflamatórios, causando os sintomas da alergia alimentar. Para que um alimento provoque reação alérgica em um indivíduo, proteínas ou outros antígenos devem ser propriamente absorvidos pelo trato gastrointestinal, interagir com o sistema imunológico, para então produzir uma resposta anormal (RAMOS, R. et al. 2013).

Já a intolerância alimentar, caracteriza-se por qualquer reação adversa a um alimento envolvendo uma resposta não imunitária. Essa pode ser de origem enzimática, farmacológica ou indeterminada. Como a intolerância à lactose, em que se desencadeiam 
sintomas pela incapacidade de digerir a lactose devido à ausência de lactase (enzima responsável pela degradação da lactose). Diferentemente da alergia, pois a intolerância não é mediada pelo sistema imunológico. Assim como as alergias, as intolerâncias alimentares apresentam variadas manifestações como: náuseas, flatulência, distensão e dor abdominal, diarreia, dor no estômago, plenitude gástrica, cefaleia, eructações, halitose, fadiga, entre outros. Elas podem compreender desde um leve desconforto até manifestações clínicas mais graves. As manifestações podem aparecer de diversas formas, incluindo manifestações cutâneas (pele e mucosa) respiratória, gastrintestinal, cardiovasculares, que podem ser de forma isolada ou combinada com manifestações cutâneas, manifestações gastrintestinais, manifestações respiratórias ou manifestações cardiovasculares. Os sintomas podem ser imediatos mediados por IgE, quando a reação ocorre em minutos ou até 8 horas da exposição do alergênico, ou pode ser tardia mediada por IgG, aparecem de 2 a 72 horas após o contato (MARTINS, A. 2015).

Os alérgenos alimentares são principalmente proteínas ou glicoproteínas com peso molecular entre 10.000 e 100.000 Daltons, alta atividade biológica, que geralmente são solúveis em água com alta estabilidade térmica e são resistentes à degradação por enzimas (proteases) e ácidos digestivos e podem, portanto, sensibilizar o indivíduo no trato gastrointestinal. Alguns ingredientes alimentares de baixo peso molecular podem se tornar alergênicos ao se ligarem às proteínas circulantes. Por outro lado, há uma diferença entre alérgenos de origem animal e alérgenos de origem vegetal: são limitados no número e na frequência de associação com fenômenos de reatividade cruzada; aqueles de origem vegetal são muito diferentes e mais frequentemente se tornam causa de reações cruzadas (JORGE, A. M. M. 2020).

A mudança para um estilo de vida urbanizado, seja como resultado do aumento do crescimento econômico ou da migração, está ligada ao desenvolvimento de alergias alimentares. Os estudos sobre migração, em particular, destacam a importante interação entre etnicidade e meio ambiente (LOH, W; TANG, ML 2018).

Apesar das limitações dos dados de prevalência disponíveis, esses estudos fornecem informações valiosas sobre a extensão do problema e os fatores de risco que estão contribuindo para o aumento da prevalência. Como acontece com a maioria das doenças crônicas, a alergia alimentar é o resultado de interações complexas entre fatores genéticos e ambientais no início da vida. Fatores de risco modificáveis e não modificáveis foram identificados no início da vida, incluindo gênero masculino, raça, genética, exposição microbiana (aumento da higiene, uso de antibióticos, exposição em cães), exposição a 
alérgenos (tempo e exposição da via de sinal, uso de antiácido) e vitamina D. O rápido aumento na prevalência de alergias alimentares nas últimas duas décadas sugere que os riscos são influenciados por fatores ambientais. Esses fatores estão ligados a alterações na flora intestinal e, portanto, podem ser atribuídos a efeitos microbianos no sistema imunológico da membrana mucosa. As limitações dos estudos que até agora examinaram a relação entre o microbioma e as alergias alimentares incluem um pequeno tamanho da amostra e / ou o uso de conscientização ou auto relato como medida de alergias alimentares (TORDESILLAS, L; BERIN, MC; SAMPSON, HA, 2017).

Na Austrália, notou-se que bebês de 12 meses com pais do Leste Asiático têm três vezes mais probabilidade de desenvolverem alergia alimentar, do que bebês de descendência não oriental. Além disso, crianças com asiáticas que nasceram na Ásia e imigraram para a Austrália tiveram um risco menor de alergia a nozes do que filhos de mães asiáticas nascidas na Austrália. A esse respeito foi relatado que oito mutações diferentes são responsáveis por $80 \%$ das mutações FLG, em Cingapura, um país com uma grande população chinesa, em comparação com apenas duas mutações nulas FLG dominantes em países europeus, junto com uma do Estudo HealthNuts, que o eczema atópico era mais provável em crianças descendentes do leste asiático, reforça a importância das interações genoma-ambiente (LOH, W; TANG, ML 2018).

Embora o leite de vaca, ovos, amendoim, nozes, peixe, marisco, trigo e soja sejam responsáveis pela maioria das reações alérgicas a alimentos na maioria dos países,diferentes localizações geográficas e hábitos alimentares têm um impacto significativo na lista dos alérgenos mais comuns em cada região. Além disso, os tipos de alimentos responsáveis por causar reações diferem, dependendo da região e da idade, grupo com alergia a vacas, leite e ovos são comuns entre as crianças mais novas, e as alergias a amendoim, nozes, peixes e crustáceos são mais comuns em crianças mais velhas e adultas. Além das alergias ao leite de vaca e ao ovo, que consistentemente são alergias, os hábitos alimentares mais comuns entre as crianças, independentemente da região geográfica, são os padrões de alergia alimentar. Na Europa e Ásia são bastante diferentes, refletindo as dietas variadas consumidas em diferentes países. Alergias a frutas devido a reações cruzadas com alergias inalantes são comuns na Europa. No norte da Tailândia, os cinco principais alimentos que causam alergia alimentar em crianças são: camarão, leite de vaca, peixe, ovo e ovos de formiga, sendo o camarão o gatilho alimentar mais comum, enquanto na Índia o grão-debico é um importante alérgeno alimentar, reflexo da dieta típica do sul da Ásia, que frequentemente inclui legumes (LOH, W; TANG, ML, 2018). 
No Brasil, os dados de prevalência de alergia alimentar são escassos e limitados a grupos populacionais, dificultando uma avaliação mais realista. Em um estudo realizado por gastroenterologistas pediátricos, a incidência de alergia a proteínas no leite de vaca foi de 2,2\%. e a prevalência de 5,4\% entre as crianças nos serviços avaliados. (SOLÉ, D., et al., 2018)

Aproximadamente $6 \%$ das crianças menores de 3 anos de idade têm AA, que geralmente é reversível com o tempo. Menos de $10 \%$ dos casos persistem na idade adulta. Nos adultos, cerca de $4 \%$ são alérgicos a pelo menos um alimento. É uma doença que pode começar cedo ou se desenvolver ao longo da vida. Os alimentos que possuem maior incidência de alergia em adultos são: amendoim, castanha, peixe ou marisco. A alergia pode se manter por toda a vida (SOLÉ, D., et al., 2018)

O leite de vaca é o alimento com maior incidência de alergia, essa alergia é quase exclusiva dos lactantes e crianças, costuma desaparecer após quatro anos e raramente é descrito na adolescência. O uso abusivo do leite de vaca como substituto do leite materno tem incidência da doença que está entre 1 e 9,5\%, tem sintoma muito variável e crianças com esse tipo de alergia podem desenvolver outros processos alérgicos como eczema e asma. Os principais alérgenos encontrados são caseína, $\alpha$-lactalbumina e $\beta$-lactoglobulina. No caso de lactentes, deve-se priorizar a manutenção do aleitamento materno exclusivo por até seis meses, sendo a alimentação complementar introduzida a partir dessa idade. Dieta de exclusão com orientação nutricional adequada para mãe e filho no momento da introdução de alimentos complementares. (SOLÉ, D., et al., 2018). O principal ponto para o tratamento das alergias ao leite de vaca é a exclusão de alimentos e seus derivados, o que implica diretamente a capacidade de identificar as diferentes proteínas do leite, muitas vezes descritas em termos pouco conhecidos pelo consumidor. Assim, deve-se enfatizar a necessidade de uma leitura cuidadosa dos rótulos dos produtos industriais, levando em consideração termos como: caseína, caseinatos, hidrolisados (de caseína, proteínas do leite e do soro de leite), lactalbumina, $\beta$-lactoglobulina, soro, creme de tratamento.

Intolerância à lactose, a lactose é o açúcar predominante no leite e também está presente em seus derivados. Sua molécula é composta de glicose e galactose, que são carboidratos menores. A quebra desse açúcar é produzida pela redução da atividade da enzima lactase, presente no revestimento intestinal, responsável pela quebra da lactose, que é posteriormente absorvida pelas células epiteliais do intestino delgado. A lactase hidrolisa a lactose em açúcar, glicose e galactose. Os sintomas mais comuns são náusea, dor abdominal, diarreia ácida em abundância, gases e mal-estar. A gravidade dos sintomas 
depende da quantidade ingerida e da quantidade de lactose que cada pessoa pode tolerar (PINTO, A; MELLO, E. 2019).

O ovo ocupa o segundo lugar no ranking das alergias alimentares mais comuns, a alergia ao ovo atinge 0,5 em crianças saudáveis, até $5 \%$ dos bebês atópicos e 50 crianças com dermatite atópica podem ser alérgicas a esse alimento. Geralmente começa na infância, na primeira vez que o alimento é oferecido. Algumas das razões que podem levar uma pessoa a desenvolver uma alergia alimentar são: herança genética, idade, hábitos alimentares, hereditariedade, exposição a alimentos, permeabilidade gastrointestinal e fatores ambientais. As alergias geralmente desaparecem com a idade. É uma reação alérgica do corpo a uma proteína encontrada na clara do ovo. As principais causas dessa alergia são as proteínas encontradas nos alvos: ovalbumina, ovomucóide (54\%) (11\%) e conalbumina (12\%). A ovalbumina pode estimular uma reação de hipersensibilidade à IgE nos alimentos e levar à liberação de mediadores de mastócitos (histamina) que atuam na pele, no nariz, nos pulmões e no trato gastrointestinal. As alterações resultantes da ação da histamina incluem aumento da permeabilidade capilar, vasodilatação, contração do músculo liso e secreção de muco. Seus sintomas podem aparecer dentro de 30 minutos ou até quatro horas após a ingestão, se a reação for imediata, mas podem ser retardados se ocorrerem por um longo período de tempo, o que pode tornar uma alergia difícil de diagnosticar (PINTO, A; MELLO, E. 2019)

As alergias ao trigo são mais comuns em comparação com outros grãos. A prevalência de alergia a outros grãos como cevada, arroz, centeio e aveia não foi estudada adequadamente. Os grãos de cereais partilham de proteínas homólogas com pólens de gramíneas e entre si, o que explica o alto índice de sensibilização desses alimentos. A alergia ao trigo se desenvolve quando o sistema imunológico se torna sensível e reage exageradamente à proteína do trigo. Nas reações alérgicas ao trigo, o inibidor da alfaamilase é um dos alérgenos mais comuns nas alergias alimentares, mediada por IgE nas frações de albumina e / ou globulina. É importante observar que a pessoa com alergia ao trigo não tem intolerância ao glúten ou doença celíaca. Em geral, as pessoas com alergia ao trigo são alérgicas a outras proteínas encontradas em outros grãos, como o centeio ou a cevada (PINTO, A; MELLO, E, 2019).

A doença celíaca é sistêmica, imunomediada e estimulada por uma reação alérgica ao glúten e suas prolaminas. Glúten ou "cola" em latim, é a proteína encontrada em grãos como aveia, cevada e centeio. A prevalência da doença celíaca é estimada em $1 \%$ da população, é uma enteropatologia inflamatória autoimune causada pela ingestão de glúten 
em indivíduos geneticamente sensíveis. Quando o glúten chega aos intestinos, ocorre uma resposta imunológica causada por um processo inflamatório crônico que resulta em danos ao revestimento do intestino delgado, causados pelo glúten. Sensibilidade ao glúten não celíaca (SGNC), que é a forma mais branda de intolerância a essa proteína. Acontece má digestão do glúten, que é composto de gliadinas e glutenina, cujos resíduos podem se alojar na parede intestinal. O tipo mais comum de trigo utilizado na alimentação é o triticum aestivum, considerado um grão de alto valor nutritivo e componente de muitos alimentos, tais como: Ex: pão, massa, pizza, sorvete, molho de shoyu, proteína vegetal hidrolisada, ketchup, amido modificado, biscoitos, balas e bebidas como cerveja. Levando em consideração termos como farelo de sêmola, gérmen de malte de sêmola de trigo ,farelo de trigo, amido de trigo hidrolisado, para a leitura dos rótulos (PINTO, A; MELLO, E. 2019).

$\mathrm{Na}$ alergia ao trigo, os principais sistemas afetados são: pele, sistema respiratório e gastrointestinal, sendo as principais manifestações pruridas: edema orofaríngeo, cólica, diarréia, e a anafilaxia, uma reação que pode ser fatal. A complexidade das reações alérgicas depende da porção do alimento consumido, do consumo simultâneo de outros alimentos, da forma como a comida é preparada, da idade do paciente, da capacidade de ingerir o alérgeno e da presença de comorbidades como dermatite atópica ou asma (PINTO, A; MELLO, E. 2019).

As reações de hipersensibilidade à ingestão de crustáceos são uma das alergias alimentares mais comuns em adultos. De acordo com a Food Allergy Research \& Education, cerca de $60 \%$ dos alérgicos a marisco tiveram sua primeira reação na idade adulta e geralmente mantinham para o resto da vida. Os crustáceos (como lagostas, caranguejos e camarões). Esses moluscos, como amêijoas e ostras são responsáveis pela maioria das reações alérgicas. Os indivíduos podem desenvolver urticária, angioedema, asma e anafilaxia. A presença de antígenos termoestáveis e termolábeis foi demonstrada em camarões. Em estudos, o alérgeno do camarão foi identificado como uma proteína muscular da tropomiosina (BRÁS, A. D. P. 2021).

A alergia à soja é a segunda alergia alimentar mais comum durante a infância $(0,4 \%$ das crianças) e ao mesmo tempo é uma das alergias com maior taxa de resolução, nomeadamente $25 \%$ aos quatro anos, $45 \%$ aos seis anos e $69 \%$ aos dez anos (BRÁS, A. D. P. 2021). A soja, de nome científico Glycine max, é uma fonte importante de proteínas de origem vegetal, de alto valor biológico pertencente à família das leguminosas. Os constituintes da soja, nomeadamente as proteínas/peptídeos, isoflavonas, que estão presentes nos grãos da soja e contêm propriedades hormonais e antioxidantes, saponinas e 
inibidores de protease trazem benefícios a nível de atividade biológica. A $\beta$-conglicinina e glicinina são as principais proteínas presentes na soja, representando 80-90\% do total de proteínas. As proteínas existentes em menor percentagem são, 2S, 9S, 15S, lectina e inibidores de protease, Todas elas trazem benefícios a nível fisiológico e nutricional. Esta alergia depende dos hábitos alimentares e da idade de introdução da soja na dieta (DE ARAÚJO, L. C. S; TORRES, S. F. R; CARVALHO, M. 2019).

A exposição a alimentos ou ingredientes provenientes de soja em pessoas com alergia a essa leguminosa dão origem a reações sistémicas. As reações são a nível cutâneo, gastrointestinal, cardiovascular, respiratório e ocorrem dentro de minutos a 2(duas) horas após a exposição ao alérgeno. (DE ARAÚJO, L. C. S; TORRES, S. F. R; CARVALHO, M. 2019). As alergias à soja podem resultar em algumas manifestações como urticária, angioedema, rinoconjuntivite, asma e até anafilaxia (BRÁS, A. D. P. 2021). Essa leguminosa pode está presente em diversos alimentos, como missô, iogurte, leite e queijo de soja, tempeh, tofu. A soja também pode ser identificada nos rótulos como: lecitina, óleo de cozinha, todos os molhos para salada prontos, proteína de soja.

A alergia ao amendoim é tipicamente mediada por IgE, com manifestação clínica ocorrendo de minutos até duas horas depois de ter ingerido o alimento. O amendoim é considerado uma leguminosa e não uma oleaginosa. Sendo possível ser alérgico a apenas alguns frutos secos ou a todos simultaneamente. Os frutos secos do amendoim podem está presentes em manteigas de frutos secos, molho pesto, bebidas vegetais, aperitivos, óleo de frutos secos. É frequente encontrá-los em pratos étnicos, como chineses, indianos ou africanos. Apesar de relatados como frequentes nos primeiros anos de vida, o início dos sintomas pode acontecer em uma minoria acima dos 20 anos de idade. Uma pesquisa americana indicou ser de 24 meses a média de idade da primeira reação ao amendoim, mais de $70 \%$ delas ocorrem logo na primeira ingestão do alimento. Crianças com alergia ao amendoim podem desenvolver sensibilização a nozes, e apresentam maior susceptibilidade a se sensibilizarem ao gergelim em comparação a crianças com alergias a outros alimentos. Por isso, é interessante investigar a sensibilização e a alergia a esses alimentos nessas crianças. Entretanto, se eses alimentos já são consumidos sem intercorrências, a investigação não é necessária (DE ARAÚJO, L. C. S; TORRES, S. F. R; CARVALHO, M. 2019). 


\subsection{Diagnóstico}

O diagnóstico de alergia alimentar deve ser baseado na análise clínica e na história Clínica. A partir das informações obtidas pela anamnese, a investigação laboratorial poderá ser implementada ou não, de exames complementares para confirmação do diagnostico, esse exame pode ser cutâneo, doseamento dos níveis séricos de IgE específicas, ou ainda pode ser feita dieta de evicção e prova de provocação oral, pois a eliminação de um ou mais alimentos da dieta pode ser útil no diagnóstico de alergia alimentar. Os testes orais podem ser mais indicados em algumas situações. O tratamento para ambas é a restrição aos alimentos que desencadeiam os sintomas (ARMINDA JORGE 2020).

O padrão atual de cuidado é evitar o alérgeno e administrar rapidamente medicamentos de resgate, como anti - histamínicos ou epinefrina, em caso de exposição acidental. E o tratamento mais promissor sob investigação é o uso de imunoterapia com antígeno-específica, que consiste no fornecimento de quantidade crescente de alérgeno por um longo período de tempo, com o objetivo de suprimir os sintomas de alergia alimentar (TORDESILLAS, L. et al., 2017).

A dieta de eliminação é a forma comprovadamente eficaz no tratamento da alergia alimentare. Uma forma de minimizar os efeitos alergênicos desses alimentos é a avaliação nutricional e a análise de cada caso, sendo essenciais para evitar o aumento da incidência de indivíduos alérgicos, principalmente quando a manifestação é na infância. Uma história clínica e exames físicos centrados na nutrição são essenciais para a garantia do adequado fornecimento de nutrientes. A proposta da intervenção nutricional é prevenir o desencadeamento dos sintomas e a piora das manifestações alérgicas, além de proporcionar a oferta devida de nutrientes para o desenvolvimento adequado e saudável do indivíduo (Silva, R. et al.2016).

\section{CONSIDERAÇÕES FINAIS}

A Alergia Alimentar é um fator cada vez mais preocupante, devido ao seu número crescente de casos, muitas vezes, fatais. A AA é mais comum em crianças e a sua prevalência parece ter aumentado nas últimas décadas em todo o mundo, estimando-se que a prevalência seja aproximadamente de $6 \%$ em menores de três anos, e de 3,5\% em adultos. A mudança para um estilo de vida urbanizado, seja como resultado do aumento do crescimento econômico ou da migração, está ligada ao desenvolvimento de alergias alimentares. As alergias alimentares são o resultado de interações complexas entre fatores 
genéticos e ambientais no início da vida $\mathrm{O}$ rápido aumento na prevalência de alergias alimentares nas últimas duas décadas sugere que os riscos são influenciados por fatores ambientais. Esses fatores estão ligados a alterações na flora intestinal e, portanto, podem ser atribuídos a efeitos microbianos no sistema imunológico da membrana mucosa. O diagnóstico de alergia alimentar deve ser baseado na análise clínica e na história Clínica. A partir das informações obtidas pela anamnese, a investigação laboratorial poderá ser implementada ou não, de exames complementares para confirmação do diagnóstico. Esse exame pode ser cutâneo, doseamento dos níveis séricos de IgE específicas. A dieta de eliminação é a forma comprovadamente eficaz nos tratamentos das alergias alimentares. $\mathrm{O}$ tratamento mais promissor que está em investigação é o uso de imunoterapia com antígenoespecífica, que consiste no fornecimento de quantidade crescente de alérgeno por um longo período de tempo, porém, há mais necessidade de estudos.

\section{REFERÊNCIA}

AGÊNCIA NACIONAL DE VIGILÂNCIA SANITÁRIA (ANVISA). Rotulagem de alimentos alergênicos. Brasília; 2017.

AMARAL, L. C. S; MOURA, R. K. V; AZEVEDO, F. H. C. (2018). Percepção dos acadêmicos de saúde portadores de alergia alimentar sobre a sua patologia. Revista Ciência \& Saberes-UniFacema, 4(1), 808-814.

ASSIS, A. A. D. (2019). Fatores alergênicos da proteína do leite e glúten e impacto na conduta nutricional e indústria de alimentos: uma revisão integrativa (Bachelor's thesis).

BERZUINO, M. B et al. (2017). Alergia alimentar e o cenário regulatório no Brasil. Revista Eletrônica de Farmácia, 14(2).

BRÁS, A. D. P. (2021). Glúten e/ou soja em medicamentos: levantamento da composição (Doctoral dissertation).

DE ARAÚJO, L. C. S; TORRES, S. F. R; CARVALHO, M. (2019). Alergias Alimentares Na Infância: Uma Revisão Da Literatura. Revista Uningá, 56(3), 29-39.

JORGE, A. M. M. (2020). Estudo de prevalência, fatores de risco e de características clínicas e serológicas de alergia alimentar em crianças dos 3 aos 11 anos, na Beira Interior.

LOH, W; TANG, M. L (2018). A epidemiologia da alergia alimentar no contexto global. Jornal internacional de pesquisa ambiental e saúde pública , 15 (9), 2043.

MARTINS, A. M. (2015). Alergias e intolerâncias alimentares (Doctoral dissertation).

PINTO, A. P. R; MELLO, E. D. D. (2019). Alergia alimentar ao trigo. International Journal of Nutrology.[Catanduva]. Vol. 12, n. 1 (2019), p. 13-17. 
PRESCOTT, S; ALLEN, K. J (2011). Alergia alimentar: cavalgando a segunda onda da epidemia de alergia. Pediatric allergy and immunology , 22 (2), 155-160

RAMOS, R. E. M; LYRA, N. R. S; DE OLIVEIRA, C. M. (2013). Alergia alimentar: reações e métodos diagnósticos. JMPHC| Journal of Management \& Primary Health Care| ISSN 2179-6750, 4(2), 54-63.

SILVA, R. L. F. D. (2016). Alergias alimentares: uma revisão integrativa com foco sobre as proteínas do leite e do ovo (Bachelor's thesis, Universidade Federal do Rio Grande do Norte).

SOLÉ D et al. Consenso Brasileiro sobre Alergia Alimentar: 2018 - Parte 1 Etiopatogenia, clínica e diagnóstico. Documento conjunto elaborado pela Sociedade Brasileira de Pediatria e Associação Brasileira de Alergia e Imunologia. Arq Asma Alerg Imunol. 2018;2(1):7-38

TORDESILLAS, L; BERIN, M. C. SAMPSON, H. A (2017). Imunologia de alergia alimentar. Immunity , 47 (1), 32-50.

\section{Como Referenciar este Artigo, conforme ABNT:}

SANTOS, M. A; MONTES, L. T. P; LOBO, F. A. T. F. Satisfação dos Usuários de Saúde Frente ao Programa Planejamento Familiar e Reprodutivo. Rev. Saúde em Foco, Teresina, v. 8, n. 3, art. 3, p. 39-53, set./dez2021.

\begin{tabular}{|l|c|c|c|}
\hline \multicolumn{1}{|c|}{ Contribuição dos Autores } & $\begin{array}{l}\text { M. A. } \\
\text { Santos }\end{array}$ & $\begin{array}{l}\text { L. T. P. } \\
\text { Montes }\end{array}$ & $\begin{array}{l}\text { F. A. T. F. } \\
\text { Lobo }\end{array}$ \\
\hline 1) concepção e planejamento. & $\mathrm{X}$ & $\mathrm{X}$ & $\mathrm{X}$ \\
\hline 2) análise e interpretação dos dados. & $\mathrm{X}$ & $\mathrm{X}$ & $\mathrm{X}$ \\
\hline 3) elaboração do rascunho ou na revisão crítica do conteúdo. & $\mathrm{X}$ & $\mathrm{X}$ & $\mathrm{X}$ \\
\hline 4) participação na aprovação da versão final do manuscrito. & $\mathrm{X}$ & $\mathrm{X}$ & $\mathrm{X}$ \\
\hline
\end{tabular}

